\title{
Heparanase and vascular endothelial growth factor expression in the progression of oral mucosal melanoma
}

\author{
ROSARIO S. RIVERA ${ }^{1,2}$, HITOSHI NAGATSUKA ${ }^{1}$, CHONG HUAT SIAR $^{3}$, MEHMET GUNDUZ $^{1}$, \\ HIDETSUGU TSUJIGIWA ${ }^{1}$, PHUU PWINT HAN ${ }^{1}$, NAOKI KATASE ${ }^{1}$, RYO TAMAMURA ${ }^{1}$, \\ KOK HAN NG ${ }^{4}$, YOSHIO NAOMOTO ${ }^{5}$, MOTOWO NAKAJIMA ${ }^{6}$ and NORIYUKI NAGAI ${ }^{1}$ \\ ${ }^{1}$ Department of Oral Pathology and Medicine, Graduate School of Medicine, Dentistry and Pharmaceutical \\ Sciences, Okayama University, Okayama City, Japan; ${ }^{2}$ University of the East, College of Dentistry, Manila, \\ Philippines; ${ }^{3}$ Department of Oral Pathology, Oral Medicine and Periodontology, Faculty of Dentistry, \\ University of Malaya; ${ }^{4}$ Unit of Stomatology, Cancer Research Centre, Institute for Medical Research, \\ Kuala Lumpur, Malaysia; ${ }^{5}$ Department of Gastroenterological Surgery, Transplant and Surgical \\ Oncology, Graduate School of Medicine, Dentistry and Pharmaceutical Sciences, Okayama University, \\ Okayama City; ${ }^{6}$ New Business and Technology Transfer, Johnson and Johnson KK, Tokyo, Japan
}

Received September 11, 2007; Accepted December 7, 2007

\begin{abstract}
Oral mucosal melanoma is an aggressive neoplasm with poor prognosis. Heparanase is an endo- $\beta-d-$ glucuronidase, which cleaves heparan sulphate chains. The vascular endothelial growth factor (VEGF) is the most potent angiogenic mitogen and interaction with its receptor (VEGFR) has been associated with angiogenesis. We investigated the expression of these molecules in the progression of oral mucosal melanoma. Immunohistochemistry was carried out in 15 oral melanotic macules and 19 oral melanomas using heparanase, VEGF, VEGFR-2, CD34 and Ki-67. Microvessel density was determined and subjected to statistical analysis. Heparanase and VEGFR-2 were not expressed in the oral melanotic macule. Atypical melanocytes and melanoma cells expressed heparanase, VEGF and VEGFR-2. An intense expression was noted in the early invasive phase, which marks the crucial transition from in situ to the invasive phase. In the invasive component, heparanase was intense but selective in the invasive fronts and at the periphery of nests unlike the extensive expression of VEGF and VEGFR-2. However, hot spots were only observed at the periphery of the nests. In conclusion, melanoma cells expressed heparanase, VEGF and VEGFR-2. The coexpression of these molecules in
\end{abstract}

Correspondence to: Dr Hitoshi Nagatsuka, Department of Oral Pathology and Medicine, Graduate School of Medicine, Dentistry and Pharmaceutical Sciences, Okayama University, 2-5-1 Shikata-cho, Okayama 700-8525, Japan

E-mail: jin@md.okayama-u.ac.jp

Key words: oral mucosal melanoma, heparanase, vascular endothelial growth factor, vascular endothelial growth factorreceptor, microvessel density atypical melanocytes and melanoma cells suggests their function in cell migration and invasion. Moreover, the intense expression in the crucial transition from in situ to the invasive phase suggests their role in the progression of the tumor. The role of VEGF and VEGFR-2 in angiogenesis was evident only at the periphery of the nests in the invasive components.

\section{Introduction}

Oral mucosal melanoma (OMM) is a rare malignant neoplasm representing $\sim 0.5 \%$ of oral malignancies and $<0.01 \%$ of all oral biopsies. OMMs upon presentation are invasive or have a mixed invasive and in situ component, while $<20 \%$ are solely in situ lesions $(1,2)$. The etiological factors and precursor lesions remain unclear due to the lack of understanding of this rare neoplasm (1). However, atypical melanocytic proliferation may be the earliest event in the transformation from the benign to the malignant stage $(2,3)$.

The transition from the in situ to the invasive phase is a crucial event in OMM progression as tumor cells migrate and invade the underlying connective tissues $(3,4)$. The sequence of events entails the production and release of enzymes indispensable for extra-cellular matrix (ECM) degradation. Heparanase (HPSE) is an endo- $\beta$-d-glucuronidase that specifically cleaves heparan sulfate (HS) chains facilitating ECM destruction, tumor cell migration and invasion (5-8). Non-enzymatic functions of HPSE have been associated with enhanced cell adhesion, migration and vascular endothelial growth factor (VEGF) induction (9-11).

The growth and proliferation of many solid tumors are mediated by the proliferation of vessels. Tumor cells produce factors that either induce or regulate angiogenesis and the balance of these factors determines the angiogenic activity (12). Among the extensive growth factors, VEGF is the only one predominantly observed at sites of angiogenesis and its 
levels correlate most closely with the spatial and temporal events of blood vessel growth (13). VEGF interacts with two high affinity transmembrane tyrosine kinase receptors, VEGFR-1 and -2, expressed by vascular endothelium. Although VEGFR-1 binds with VEGF with a higher affinity, it is believed to act as a decoy receptor by modulating the availability of VEGF to VEGFR-2, the principal receptor for VEGF signaling $(14,15)$.

This study aimed to investigate the heterogeneity in the expression of HPSE, VEGF and VEGFR-2 in relation to the migration, invasion and progression of OMM. The relative increase in the expression of these molecules correlated with the progression, suggesting their roles in the unparalleled aggressive behavior of OMM.

\section{Materials and methods}

Tissue specimens. Fifteen oral melanotic macules and 19 OMMs were retrospectively analyzed. OMMs were diagnosed as primary OMM based on a thorough and complete case history and physical examination to confirm that the patients had no occult melanotic lesions in other parts of the body. Representative paraffin blocks were meticulously chosen and sectioned into $3-\mu$ pieces. Tissue specimens included normal tissues and some atypical melanocytic proliferations. OMM cases were categorized as 9 invasive, 9 invasive with an in situ component and 1 in situ (2). Areas observed in OMM included atypical melanocytic proliferation, an early invasive component (in situ component depicting early invasion in the connective tissues) and an invasive component (3). The cases were $>4 \mathrm{~mm}$ in thickness except for the purely in situ OMM. The experimental ethics and review committee of our institution approved the study protocol.

Immunohistochemistry. Deparaffinized tissue sections were blocked for endogenous peroxidase activity using $3 \%$ hydrogen peroxide in methanol for $30 \mathrm{~min}$, washed with trisbuffered saline solution and treated for antigen retrieval. For heat treatment (HPSE and VEGF), the slides were immersed in citrate buffer ( $\mathrm{pH} \mathrm{6.0)}$ ) and allowed to boil for $1 \mathrm{~min}$. For heat pressure treatment (VEGFR-2 and Ki-67), the slides were immersed in citrate buffer ( $\mathrm{pH}$ 6.0) and placed in an autoclave for $5 \mathrm{~min}$ at $121^{\circ} \mathrm{C}$. For trypsin treatment (S100 and CD34), the slides were immersed in $0.1 \%$ pre-warmed trypsin solution for $15 \mathrm{~min}$ at $37^{\circ} \mathrm{C}$. The slides were covered initially for $15 \mathrm{~min}$ with 5\% normal mouse serum-blocking solution or serum-free protein block, followed by a primary antibody and then incubated overnight at $4^{\circ} \mathrm{C}$. All antibodies used were commercially available except for HPSE and the specificity of the antibody have previously been characterized and reported (16).

Immunoreactions were performed using a Vectastain peroxidase ABC kit (Vector Laboratories, Burlingame, CA, USA) or with Envision ${ }^{\mathrm{TM}}$ detection reagent peroxidase (DakoCytomation, Carpinteria, USA) and a 3-aminoethylcarbazole (AEC) substrate chromogen (DakoCytomation) was used as a detection reagent. Primary antibodies were replaced with normal mouse serum for negative control. Table I shows the antibodies used with their corresponding dilutions.
Table I. Antibodies used.

\begin{tabular}{ll}
\hline Antibody & Dilution \\
\hline HPSE & $1: 2000$ \\
VEGF (Santa Cruz) & $1: 150$ \\
VEGFR-2 (Santa Cruz) & $1: 100$ \\
S100 (Nichirei) & RTU $^{\mathrm{a}}$ \\
CD34 (Nichirei) & RTU \\
Ki-67 (Dako) & $1: 100$ \\
\hline
\end{tabular}

${ }^{\text {a Ready to use. }}$

Table II. A summary of HPSE, VEGF and VEGFR-2 expression.

Tissue samples HPSE VEGF VEGFR-2

\begin{tabular}{lccc}
\hline Oral melanotic macule & - & \pm & - \\
Atypical melanocytic proliferation & + & + & + \\
In situ component & + & + & + \\
Early invasive component & ++ & ++ & ++ \\
Invasive component & & & \\
$\quad$ Center of nest & \pm & ++ & ++ \\
Periphery of nest & ++ & ++ & ++
\end{tabular}

(-) Negative, $( \pm)$ focally positive, $(+)$ moderately positive and (++) extensively positive expression by melanocytes, atypical melanocytes or melanoma cells.

Immunoreaction was regarded as negative (-) when no expression was observed; focally positive $( \pm)$ when $<20 \%$ of melanocytes, atypical melanocytes or melanoma cells were positive and the intensity was weak; moderately positive (+) when $20-50 \%$ were positive and the intensity was moderate and extensively positive $(++)$ when $>50 \%$ were positive and the intensity was strong (Table II).

Microvessel density (MVD). The three most vascular areas (corresponding to hot spots), positive to CD34, were selected under scanning magnification and counted under a $20 \mathrm{x}$ objective lens with an etched square graticule inserted in the ocular $(17,18)$. The mean MVDs were calculated and evaluated using Student's t-test. A P-value of $<0.05$ was considered significant. All calculations were computer-based (SPSS 11.5, SPSS Inc., Chicago, IL, USA).

\section{Results}

No difference in the expression of HPSE, VEGF and VEGFR-2 was observed among the cases which were $>4 \mathrm{~mm}$ in thickness, some with bone invasion and regional metastasis. Heterogeneity in the expression was noted in the different stages of progression and in the architecture of the nests of the invasive component. 

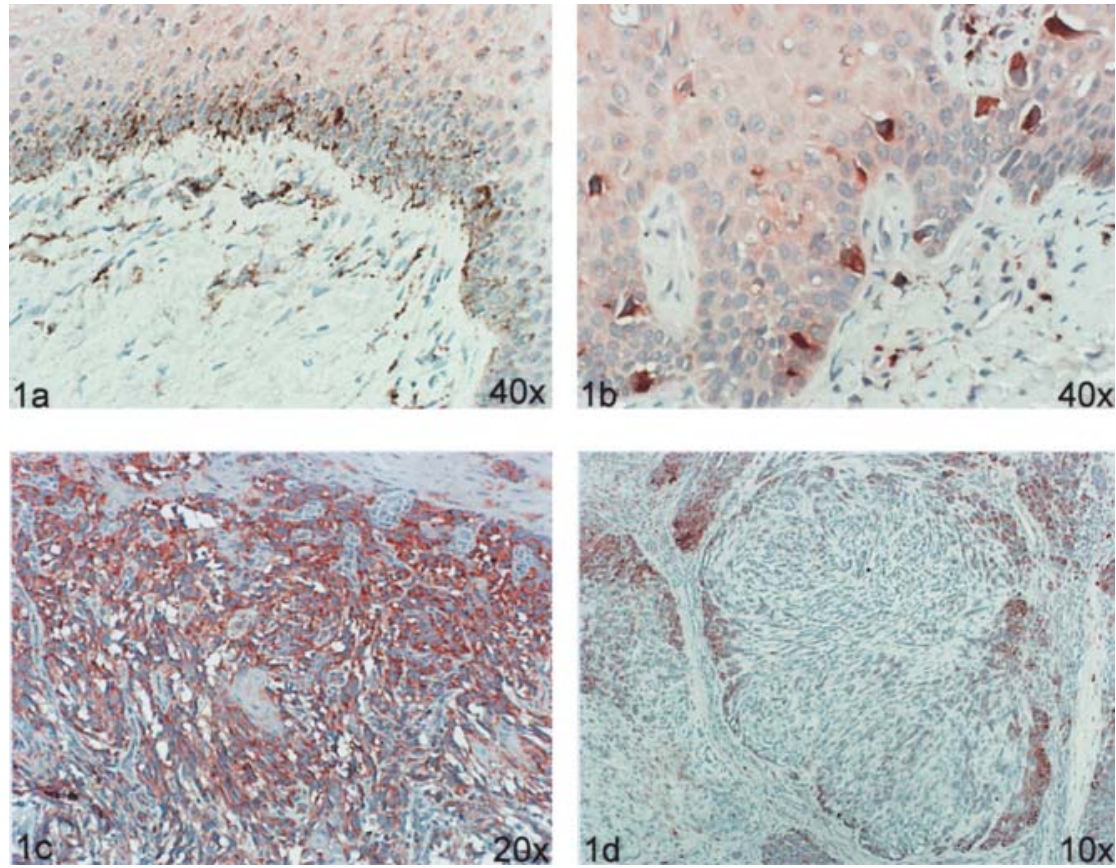

Figure 1. HPSE expression. Melanocytes in the oral melanotic macule did not express HPSE (1a) but the atypical melanocytes did (1b). Intense expression of HPSE by invading melanoma cells in the early invasive component (1c). HPSE was mostly expressed by melanoma cells at the periphery of the nests (1d).
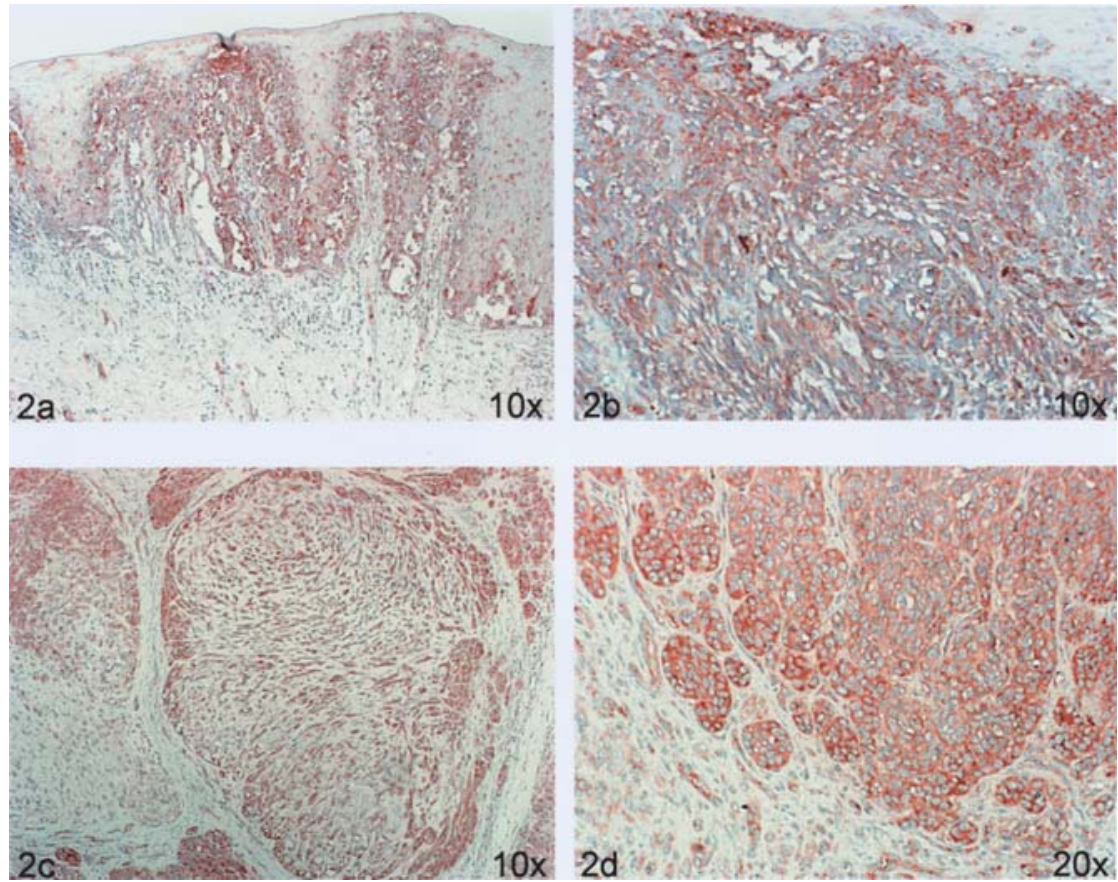

Figure 2. VEGF and VEGFR-2 expression. Intense VEGF expression in situ (2a) and the early invasive component (2b). Extensive expression of VEGFR-2 in the invasive components $(2 \mathrm{c}$ and $\mathrm{d})$.

HPSE expression. Melanocytes at the basal layer in the oral macule were disclosed by S100 protein. These cells did not express HPSE (Fig. 1a). Expression was detected in atypical melanocytes (Fig. 1b) and an increased intensity was detected in melanoma cells in the early invasive (Fig. 1c) and invasive components. However, HPSE was localized at the periphery of nests (Fig. 1d), invasive fronts, individually proliferating and invading tumor cells and those adjacent to blood vessels. In deep areas, fibroblasts also expressed HPSE.

VEGF and VEGFR-2 expression. VEGF was expressed by a few melanocytes in the oral melanotic macule. However, the atypical melanocytes expressed VEGF and VEGFR-2. The expression was detected in the in situ component (Fig. 2a) but 

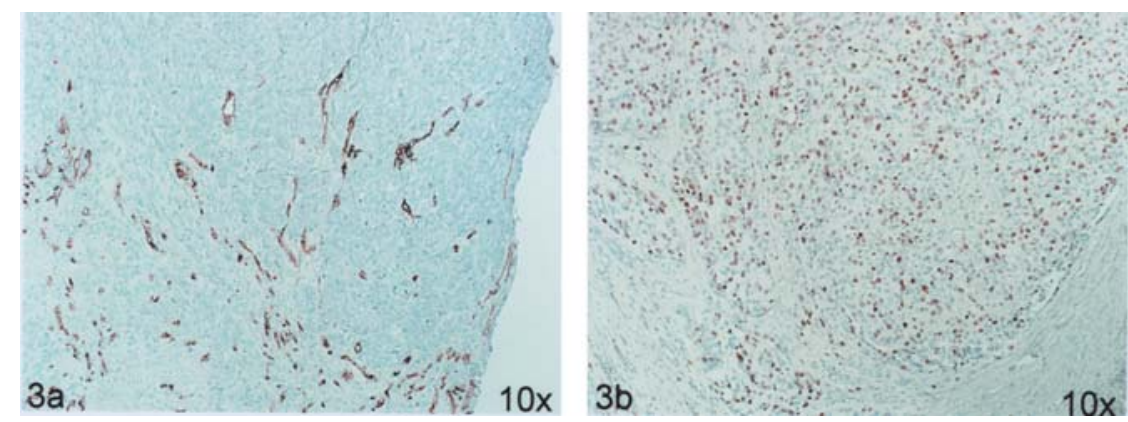

Figure 3. CD34 and Ki-67. Vessels expressing CD34 in the invasive component (3a). High proliferation activity exemplified by Ki-67 (3b).

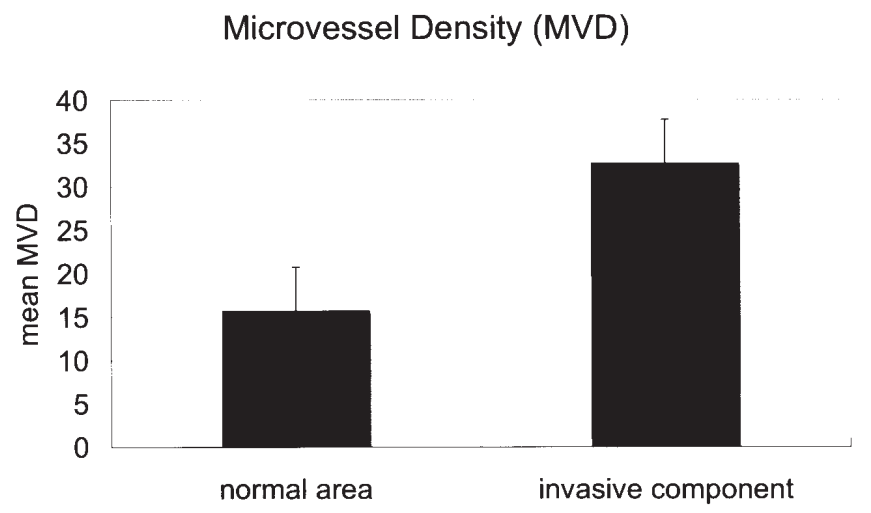

Figure 4. Microvessel density. An increase in the mean MVD was observed in the invasive OMM compared to the normal areas $(\mathrm{P}=0.0049)$.

stronger intensities were observed in the early invasive (Fig. 2b) and invasive components (Fig. 2c and d). Endothelial cells, especially those that were adjacent to the tumor masses, also expressed VEGFR-2.

CD34 and MVD. Vessels were mostly concentrated at the periphery of the nests and infrequently in the center (Fig. 3a). To check the proliferation activity, Ki-67 was used. Ki-67 was widely expressed by melanoma cells (Fig. 3b). The mean MVD varied widely, ranging from 11.33 to 73.67 . The mean MVD showed an increase in the vessel count from the normal areas to the invasive component with a significant $\mathrm{P}$-value of $0.0049(\mathrm{P}<0.05)$ (Fig. 4).

\section{Discussion}

Although a rare tumor, the unique biological behavior of OMM, different from its cutaneous counterpart and its poor prognosis, justifies the necessity for a further understanding of the mechanism behind its aggressiveness. Melanoma cells secrete a plethora of molecules essential for invasion, growth and proliferation. This study demonstrated the change in the expression of HPSE, VEGF and VEGFR-2 by the melanoma cells in the course of progression.

The expression of VEGF by the melanocytes in the oral melanotic macule may be a usual occurrence in the benign lesions (19). However, the expression of HPSE, VEGF and VEGFR-2 in the atypical melanocytes suggests that these molecules are probably concerned with the earliest event of transformation from the benign to malignant stage. The ability of the atypical melanocytes to migrate laterally and in a superior manner within the epithelium was probably related to the expression of these molecules $(9,10,14)$.

The expression of HPSE, VEGF and VEGFR-2 was highly noted in the early invasive phase, which is a crucial stage of transformation from in situ to the invasive phase. The change in immunoreaction was not only based on the increase in the number of cells that expressed the molecules but also on the intensity of the expression. The enzymatic ability of HPSE to cleave HS chains leading to a loss of BM integrity and invasion of the ECM indicates its involvement in the early progression at the primary tumor site (20). VEGF/VEGFR-2 expression has also been observed during the transition from the horizontal to vertical growth phase which promoted the invasion of melanoma cells as well as other carcinoma cell lines $(4,14,21)$. The overall results suggest that HPSE, VEGF and VEGFR-2 expression contributes to the invasiveness of melanoma cells.

Heterogeneity was observed in the invasive phase. The intense HPSE expression was limited at the periphery of the nests suggesting the primary role of HPSE in tumor invasion. On the other hand, VEGF and VEGFR-2 expression was extensive not only in hot spots (periphery of the nests) but also in areas devoid of vessels. Our results suggest that VEGF and VEGFR-2 expression correlated with angiogenesis, as attested by the tremendous increase in MVD. However, the expression of VEGF and VEGFR-2 at the center of the nests suggests another function aside from angiogenesis. The autocrine action of VEGF and VEGFR-2 may play a role in vasculogenic mimicry where tumor cells acquire the endothelial cell phenotype (22). This is a possible mechanism for the viability of the melanoma cells in a nutrition-deficient environment (22-25).

OMM invasion, growth and proliferation are complex processes involving various enzymes, cytokines and growth factors. However, HPSE and VEGF/VEGFR-2 interactions may not be the sole regulators of OMM aggressive behavior, as they may have essential and significant contributions in OMM tumorigenesis. A clearer understanding of the mechanism behind the aggressiveness of OMM could lead to therapies that may block the carcinogenic process especially during the early stage and keep local disease under control.

In conclusion, OMM cells expressed HPSE, VEGF and VEGFR-2. The coexpression of these molecules in atypical 
melanocytes and melanoma cells suggests their function in cell migration and invasion. The intense expression in the crucial transition from in situ to the invasive phase suggests their role in the progression of the tumor. The role of VEGF and VEGFR-2 in angiogenesis was evident only at the periphery in the nests of the invasive components.

\section{Acknowledgements}

This work was supported in part by grants-in-aid for scientific research from the Ministry of Education, Culture, Sports, Science and Technology (B17406027). The authors would also like to thank Ms. Kazuko Funakoshi for her technical expertise and Mr. Beyhan Cengiz for the statistical analysis.

\section{References}

1. Hicks MJ and Flaitz CM: Oral mucosal melanoma: epidemiology and pathobiology. Oral Oncol 36: 152-169, 2000.

2. Barker BF, Carpenter WM, Daniels TE, et al: Oral mucosal melanomas: the WESTOP Banff workshop proceedings. Western Society of Teachers of Oral Pathology. Oral Surg Oral Med Oral Pathol Oral Radiol Endod 83: 672-679, 1997.

3. Nagatsuka H, Rivera RS, Gunduz M, et al: Immunolocalization and distribution patterns of type IV collagen alpha chains in oral mucosal melanoma. Virchows Arch 447: 710-716, 2005.

4. Erhard H, Rietveld FJ, van Altena MC, Brocker EB, Ruiter DJ and de Waal RM: Transition of horizontal to vertical growth phase melanoma is accompanied by the induction of vascular endothelial growth factor expression and angiogenesis. Melanoma Res 7: S19-S26, 1997.

5. Nakajima M, Irimura T and Nicolson GL: Heparanases and tumor metastasis. J Cell Biochem 36: 157-167, 1988.

6. Vlodavsky I and Friedmann Y: Molecular properties and involvement of heparanase in cancer metastasis and angiogenesis. J Clin Invest 108: 341-347, 2001.

7. Parish CR, Freeman C and Hulett MD: Heparanase: a key enzyme involved in cell invasion. Biochim Biophys Acta 1471: M99-M108, 2001.

8. Elkin M, Ilan N, Ishai-Michaeli R, Friedmann Y, Papo O, Pecker I and Vlodavsky I: Heparanase as mediator of angiogenesis: mode of action. FASEB J 15: 1661-1663, 2001.

9. Gingis-Velitski S, Zetser A, Flugelman MY, Vlodavsky I and Ilan N: Heparanase induces endothelial cell migration via protein kinase B/Akt activation. J Biol Chem 279: 23536-23541, 2004.

10. Goldshmidt O, Zcharia E, Cohen M, et al: Heparanase mediates cell adhesion independent of its enzymatic activity. FASEB J 17: 1015-1025, 2003.
11. Zetser A, Bashenko Y, Edovitsky E, Levy-Adam F, Vlodavsky I and Ilan N: Heparanase induces vascular endothelial growth factor expression: correlation with p38 phosphorylation levels and Src activation. Cancer Res 66: 1455-1463, 2006.

12. Folkman J and Shing Y: Angiogenesis. J Biol Chem 267: 10931-10934, 1992.

13. Robinson CJ and Stringer SE: The splice variants of vascular endothelial growth factor (VEGF) and their receptors. J Cell Sci 114: 853-865, 2001

14. Lacal PM, Ruffini F, Pagani E and D'Atri S: An autocrine loop directed by the vascular endothelial growth factor promotes invasiveness of human melanoma cells. Int J Oncol 27: 1625-1632, 2005.

15. Shalaby F, Rossant J, Yamaguchi TP, Gertsenstein M, Wu XF, Breitman ML and Schuh AC: Failure of blood-island formation and vasculogenesis in Flk-1-deficient mice. Nature 376: 62-66, 1995.

16. Toyoshima M and Nakajima M: Human heparanase. Purification, characterization, cloning, and expression. J Biol Chem 274: 24153-24160, 1999

17. Foss AJ, Alexander RA, Jefferies LW, Hungerford JL, Harris AL and Lightman S: Microvessel count predicts survival in uveal melanoma. Cancer Res 56: 2900-2903, 1996.

18. Kato T, Kimura R, Ishii N, Fujii A, Yamamoto K and Kameoka S: The methodology of quantitation of microvessel density and prognostic value of neovascularization associated with long-term survival in Japanese patients with breast cancer. Breast Cancer Res Treat 53: 19-31, 1999.

19. Pisacane AM and Risio M: VEGF and VEGFR-2 immunohistochemistry in human melanocytic naevi and cutaneous melanomas. Melanoma Res 15: 39-43, 2005.

20. Mikami S, Ohashi K, Katsube K, Nemoto T, Nakajima M and Okada Y: Coexpression of heparanase, basic fibroblast growth factor and vascular endothelial growth factor in human esophageal carcinomas. Pathol Int 54: 556-563, 2004.

21. de Jong JS, van Diest PJ, Michalides RJ, van der Valk P, Meijer CJ and Baak JP: Expression of growth factors, growthinhibiting factors, and their receptors in invasive breast cancer. II: Correlations with proliferation and angiogenesis. J Pathol 184: 53-57, 1998.

22. Maniotis AJ, Folberg R, Hess A, et al: Vascular channel formation by human melanoma cells in vivo and in vitro: vasculogenic mimicry. Am J Pathol 155: 739-752, 1999.

23. Dome B, Paku S, Somlai B and Timar J: Vascularization of cutaneous melanoma involves vessel co-option and has clinical significance. J Pathol 197: 355-362, 2002.

24. Zhang S, Guo H, Zhang D, Zhang W, Zhao X, Ren Z and Sun B: Microcirculation patterns in different stages of melanoma growth. Oncol Rep 15: 15-20, 2006.

25. Lee YJ, Nagai N, Siar CH, et al: Angioarchitecture of primary oral malignant melanomas. J Histochem Cytochem 50: 1555-1562, 2002. 